\title{
As $E$. coli continues to claim lives, new approaches offer hope
}

On 25 May-just as the deadliest outbreak of Escherichia coli on record was beginning to tear through Germany-a team of physicians happened to publish an experimental therapy that could save lives in future outbreaks of this kind. The article described how an antibody therapy called Soliris (eculizumab) had successfully reversed the kidney damage and neurological symptoms seen in three young E. coli-infected children suffering from hemolytic-uremic syndrome (HUS), a deadly complication also seen in many victims of the German outbreak (N. Engl. J. Med. doi:10.1056/ NEJMc1100859, 2011).

Soliris has been on the market since 2007 to treat a rare blood disorder called paroxysmal nocturnal hemoglobinuria, and it costs a staggering $\$ 400,000$ per year. The antibody's manufacturer, Connecticut-based Alexion Pharmaceuticals, had been testing Soliris in people who develop HUS without any E. coli infection. It filed for regulatory approval for this indication in the US and Europe earlier this year and reported further positive clinical trial data at last month's Congress of the European Hematology Association in London.

But spurred by requests from German physicians after the findings related to $E$. coli-induced HUS came out, starting on 30 May, Alexion worked with health authorities in Germany to dispense the drug on a compassionate-use basis at no charge.

Since then, doctors across the country have administered Soliris to hundreds of those affected by the outbreak. And on 20 June, Alexion formally launched an open-label clinical trial in people sickened by E. coli who have received Soliris during the crisis. "A clinical trial is the best environment to ensure that the drug is administered in a controlled manner to support safety and potential efficacy," says Irving Adler, an Alexion spokesperson. But, according to Franz Schaefer, the lead author of the initial study and a nephrologist at the Center for Pediatrics and Adolescent Medicine in Heidelberg, Germany, the numbers coming in suggest that not everyone has responded as well as the kids in his team's study. "The response, as far as I've heard, is kind of mixed," he says.

Unfortunately, when it comes to the varieties of $E$. coli that produce shiga toxins, such as those seen in Germany, few treatment options exist. Antibiotics, for example, can further stimulate the release of these toxins, making HUS symptoms worse, but no drugs are licensed specifically to treat the disease. Instead, physicians commonly perform plasmapheresis, replacing people's own blood fluids with those

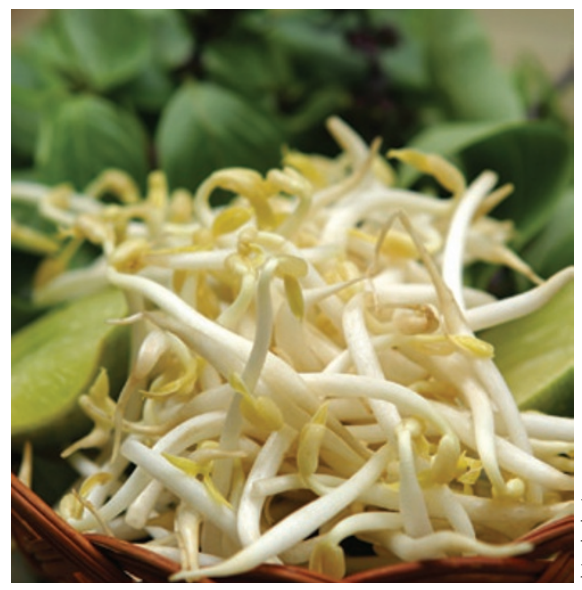

Sprouting up: New treatments target outbreak.

taken from a donor to rid the body of the bacterial toxins.

"Plasmapheresis is a potentially effective treatment, and then on top of it you can give eculizumab," notes Jochen Reiser, head of nephrology at the University of Miami School of Medicine. "But there's really nothing else. We don't really have anything more specific than that." However, several experimental drugs are in development.

The furthest along is Thallion Pharmaceuticals' Shigamabs, a dual-antibody product designed to specifically neutralize the two types of shiga toxin secreted by pathogenic E. coli well before HUS manifests. Soliris, by comparison, is given after HUS has already developed and works by neutralizing immune molecules that contribute to the inflammation and blood clotting seen in the disease.

"We don't think that the antitoxin can rescue someone who already has full-blown HUS, but we think it can prevent HUS from ever happening if it's given early enough in the disease process," says Alison O'Brien, a microbiologist at the Uniformed Services University of the Health Sciences in Bethesda, Maryland who helped develop the product.

\section{It takes two}

Montreal-based Thallion already has good safety data for its antibody combo from phase 1 studies in adults and the first half of its phase 2 trial involving 22 children and adolescents in South America. On 30 May, the company announced that it had also enrolled the first of another 21 participants to examine a higher dose of the product. At the same time, as Nature Medicine went to press, the company's chief executive Allan Mandelzys was in talks with European health authorities to get the product into the hands of physicians there in the immediate future. "We wanted to update them on our current status of development and inquire if there was anything we could do to help," he says.

Antibodies are not the only experimental approach to combating HUS-associated E. coli, though. Earlier this year, for example, researchers at the Universities of Buffalo and New Mexico showed that administering zincbased salts to $E$. coli-infected rabbits lowered toxin levels and decreased the amount of bacteria-induced tissue damage in the intestine (Infect. Immun. 79, 1696-1705, 2011). And in May, researchers from the Boston University School of Medicine reported that injecting a small peptide molecule that can enter cells and neutralize shiga toxin there reduced disease severity in juvenile baboons (Pediatr. Nephrol. doi:10.1007/s00467-011-1913-y, 2011).

Meanwhile, another study that same month showed that feeding a small-molecule drug called C-9-first developed by the Cambridge, Massachusetts biotech Genzyme to treat people with rare metabolic syndromes-to E. coli-infected rats cut the death rate in half and reduced the extent of kidney and intestinal injury in the surviving animals (Pediatr. Res. 69, 390-394, 2011). "Only one to two days of treatment with this compound is enough to avoid the cytotoxic effects of the shiga toxin," says lead author Claudia Silberstein of the University of Buenos Aires in Argentina.

Some researchers are hoping to stop E. coli even further up the tracks. Debora Barnett Foster, a cellular microbiologist at Ryerson University in Toronto, is working on a vegetable spray designed to expose the microbe's vulnerabilities the moment it hits the gastric system. Reporting online in March, she showed that a particular peptide impaired the ability of shiga-producing E. coli to survive in a lab dish under acidic conditions that mimicked those found in the human gastric system (Microbiology 157, 1768-1775, 2011). "If these bacteria saw this peptide prior to seeing acid stress, we could compromise their ability to repair their DNA," she says.

All these various approaches, researchers agree, should help better prepare health officials the next time that shiga toxin-producing $E$. coli strikes. For people sick during the ongoing German outbreak, that day can't come soon enough. "Honestly," says O’Brien, "we feel that if [Shigamabs] had been used when people starting coming in with bloody diarrhea, the HUS rate would have come down."

Elie Dolgin 